\title{
A agregação das Tecnologias de Informação e Comunicação ao espaço público urbano: reflexões em torno do Projeto CyberParks - COST TU 1306
}

\author{
The penetration of Information and Communications Technologies into public \\ spaces: some reflections from the Project CyberParks - COST TU 1306
}

Carlos Smaniotto Costa ${ }^{[0]}$, Marluci Menezes ${ }^{[b]}$

[a] Universidade Lusófona, Centro de Estudos Interdisciplinares em Educação e Desenvolvimento (CeiED), Lisboa, Portugal

${ }^{[b]}$ Laboratório Nacional de Engenharia Civil (LNEC), Lisboa, Portugal

\section{Resumo}

Apresenta-se o Projeto CyberParks para discutir a relação entre o uso de meios digitais (smartphones, conexões wi-fi etc.) em espaços públicos (parques, jardins, praças etc.) e a contribuição das novas tecnologias para qualificar e aumentar a participação de usuários na sua produção e manutenção. CyberParks é guiado pelas questões: (1) Como as Tecnologias de Informação e Comunicação (TIC) afetam o uso do espaço público? (2) Quais são os riscos associados à recente, mas acelerada, dinâmica de difusão das TIC no espaço urbano? (3) Como os usos identificados se enquadram no conceito de sociedade virtual? Em suma, trata-se de investigar a contribuição das TIC na transformação das cidades em contextos mais inclusivos, e não somente mais high tech. 0 projeto objetiva desvendar o papel das TIC na produção e no desenho de espaços híbridos. Uma premissa é aumentar o conhecimento das possibilidades que as TIC abrem para uma melhor compreensão da forma como as pessoas usam esses espaços. Discutem-se o âmbito e a pertinência da perspectiva de estudo focada na relação entre TIC e espaço público, apresentando objetivos do Projeto CyberParks. Em sequência, problematizam-se algumas das principais linhas de investigação, apresentando os resultados de um estudo de caso desenvolvido em Lisboa, Portugal.

Palavras-chave: Espaços públicos abertos. Tecnologias de Informação e Comunicação. Realidade aumentada e a cidade. Desenho urbano. Paisagem urbana.

\section{Abstract}

In this paper we use the European research project CyberParks to discuss the relationship between digital media (smart phones, tablets, wi-fi network) and its use in public open spaces (parks, gardens, squares, etc.) and the contribution of the emerging technologies to improve the quality, production, and maintenance of open spaces through more extensive participation by users. The leading questions of CyberParks are: (1) how ICT affect the use of public space; (2) what are the risks associated with an increasing aggregation of ICT into public spaces; and (3) how new uses contribute to a virtual society concept. In summary: how ICT transform our cities into more inclusive places, rather than just higher tech. The projects seeks to better understand the role of ICT in the production and design of public spaces, and what possibilities they open to increase the understanding of

CSC é professor, Doutor em Planejamento urbano e ambiental, e-mail: smaniotto.costa@ulusofona.pt MM é investigadora, Doutora em Antropologia, e-mail: marluci@lnec.pt 
how people use public spaces. The reflection discusses the scope and relevance of the relationship between ICT and public space, followed by the key features and objectives of the CyberParks Project. In sequence, some of the main research lines are discussed from the conceptual framework that guides the project, to finally discuss some preliminary results of a case study conducted in Lisbon.

Keywords: Open public space. Information and Communication Technology. Digitally-augmented city. Urban design. Urban landscape.

\section{A pertinência do tema}

A utilização das tecnologias de informação e comunicação (TIC) manifesta-se, de modo geral, em uma vasta gama de atividades sociais e econômicas, decorrendo daí um profundo e duradouro impacto nas nossas vidas. As chamadas tecnologias emergentes estão mudando o nosso comportamento, o modo como nos relacionamos e comunicamos, a forma como utilizamos e nos apropriamos da cidade, bem como tendem a alterar e a tornar mais complexas as necessidades sociais e de infraestrutura urbana.

Se, em um dia ensolarado, podemos observar pessoas em parques aproveitando para relaxar e também recorrendo a smartphones, tablets e notebooks, paralelamente observa-se a existência de um grande número de pessoas que preferem permanecer nos espaços domésticos ou interiores. 0 wired way of life, indubitavelmente, atrai mais e mais as pessoas a utilizar no seu cotidiano as comodidades oferecidas pelas novas tecnologias, seja no contexto de trabalho, educação ou lazer. No entanto, esse novo caminho tecnológico não necessariamente significa o aumento da apetência social para estar ao ar livre e usar os espaços públicos, ainda que, de algum modo, essas pessoas possam estar vivenciando a esfera pública a partir de uma experiência de realidade virtual e/ou de navegação pela comunidade virtual. A vida sedentária enquanto opção por não usufruir dos espaços ao ar livre tem aspectos negativos para a saúde e o bem-estar físico e mental, tanto individual como coletivamente (Godbey, 2009; Thompson, 2002), assim como para o próprio ambiente urbano, pois a sua qualidade, vitalidade e segurança dependem da apropriação desses espaços pelas pessoas, que são quem lhes dão vida. São muitos e diferenciados os aspectos que influenciam tanto o uso de espaços públicos (e a sua frequência) quanto a tendência para ficar em casa, tais como as questões do conforto ambiental, da segurança e também da cultura urbana. É certo ainda considerar que, na conjuntura atual, as TIC detêm um importante papel nas novas tendências de uso e de apropriação da cidade. Mas até que ponto a mudança de comportamento, associada ao crescente e recorrente uso das tecnologias digitais, altera a maneira como usamos e apreciamos as nossas praças, jardins e parques?

Hampton et al. (2009) abordaram a questão das mudanças de comportamento e nas formas de interação social provocadas pela era digital, observando a emergência de novos estilos de vida. E, nesse sentido, Graham \& Aurigi preconizaram, já em 1997, a relação entre crise do espaço público, polarização social e fosso digital.

As novas dinâmicas sociais permitem questionar as conexões entre a cidade real e o espaço virtual, já que o uso contemporâneo das TIC tem reflexo nas formas de uso e de apropriação da cidade, fazendo emergir novas necessidades, anseios e perspectivas, sobretudo tendo em consideração uma sociedade urbana cada vez mais pluralista. Como, então, projetar espaços públicos para essa sociedade? Como as TIC podem contribuir com o processo de produção de espaços públicos mais inclusivos e híbridos? Interessa-nos aprofundar o conhecimento sobre a relação entre os espaços e os seus utilizadores para, assim, formular recomendações e estratégias que contribuam para melhorar o ambiente urbano. Esse é o desafio proposto pelo Projeto CyberParks.

\section{O Projeto CyberParks}

Como podemos capitalizar o nosso recente relacionamento com a vida digital para encorajar mais pessoas a sair de casa, a adotar um estilo de vida mais ativo e a usar mais intensivamente os espaços públicos urbanos? Que oportunidades as tecnologias emergentes abrem para quem se interessa pela produção e pelo uso de espaços públicos? Essas são perguntas centrais que o Projeto CyberParks - Fostering 
knowledge about the relationship between Information and Communication Technologies and Public Spaces supported by strategies to improve their use and attractiveness - propõe desvendar.

O projeto é financiado pela União Europeia (2014-2018) por meio de um programa de incentivo à investigação chamado COST Framework (2015) e é coordenado pelo Laboratório Experimental de Educação, Comunicação e Espaço Público do Centro de Estudos Interdisciplinares em Educação e Desenvolvimento (CeiED), da Universidade Lusófona de Lisboa, Portugal. 0 projeto conta, atualmente, com parceiros de 28 países europeus e de Israel, abrangendo 80 pesquisadores de várias disciplinas, tais como desenho e planejamento urbano, educação, comunicação, sociologia, antropologia, psicologia, saúde pública, direito urbanístico, arte pública, informática, bem como investigadores no domínio das TIC e programadores, novas tecnologias e fomento econômico.

CyberParks entende-se como uma plataforma de investigação que explora as relações entre as novas tecnologias, a produção e o uso do espaço público, e a sua importância para um desenvolvimento urbano sustentável. 0 projeto é desenvolvido a partir de cinco grupos transdisciplinares de trabalho: (1) metodologias digitais, (2) etnografia urbana, (3) reflexão conceitual, (4) criação de um CyberParks e (5) expansão da rede e difusão de conhecimentos. Mais informações sobre o projeto, participantes e resultados estão disponíveis nos sites do programa COST e do próprio projeto.

A investigação e o compartilhamento de experiências centram-se na forma como as TIC e os novos dispositivos digitais podem contribuir para os seguintes aspectos:

- Atrair mais utilizadores para interagir nos espaços públicos;

- Criar espaços públicos mais inclusivos, visando à melhoria da condição física e mental dos utilizadores e o bem-estar das pessoas;

- Desenvolver novas metodologias de investigação que amparem o planejamento, a produção e o uso dos espaços públicos.

Com a criação da plataforma CyberParks, aberta a todos que queiram participar das discussões on-line, o projeto fomenta a cooperação transversal e interdisciplinar no sentido de associar e de incrementar o conhecimento disponível em diferentes áreas. Ao explorar o entrelaçamento de diferentes perspectivas, o projeto garante, assim, que não só as possíveis e necessárias interligações entre dispositivos digitais, espaços públicos urbanos, estilos de vida e necessidades da população sejam levados em consideração, mas também a detecção de práticas sociais que sejam inovadoras. Isso, a longo prazo, permitirá contribuir para a definição de um panorama mais amplo sobre as implicações, oportunidades e desafios que a fusão e a imersão dos mundos real e virtual representam.

Propõe-se, assim, explorar tópicos relacionados com o futuro desenvolvimento das TIC para efeitos de reconhecimento dos valores dos espaços públicos urbanos, identificação de estilos de vida contemporâneos, previsão de futuras necessidades, de modo a identificar os desafios que se colocam em âmbito do desenvolvimento de melhores espaços urbanos e de cidades mais inclusivas e participativas. Para efeito, o projeto está realizando estudos de caso em diversas cidades europeias, tais como Amsterdã (Holanda), Barcelona (Espanha), Lisboa (Portugal), Ljubljana (Eslovênia), Valletta (Malta) e Sófia (Bulgária). Nesse sentido, o objetivo é obter uma visão mais abrangente sobre um tema emergente.

\section{As Tecnologias de Informação e Comunicação (TIC)}

Por TIC entende-se os procedimentos, métodos, equipamentos e recursos para processar informação e proceder a diferentes formas de comunicação via suporte tecnológico e de dispositivos digitais, o que inclui a internet (por redes e sem fio), tablets, smartphones etc. Esses novos dispositivos não só viabilizam a comunicação virtual como também contribuem para, em tempo real, motivar as pessoas a compartilhar interesses, ideias, opiniões etc., independentemente de uma posição geográfica ou da presença física.

Em termos de urbanismo, os recursos disponibilizados pelas TIC possibilitam a coleta de informações sobre espaços específicos, equipamentos e serviços que as pessoas utilizam, bem como contribuem para o compartilhamento de informação sobre lugares reais, colaborando não só para a construção de espaços virtuais, algo que não é possível sem essas tecnologias, mas, sobretudo, para uma interação entre mundo real e virtual (Smaniotto Costa \& Schmitz, 2013).

A difusão da internet e a possibilidade de se comunicar on-line impulsionaram significativamente 
o crescimento da mídia digital e de redes sociais, como facebook. Essas mídias sociais tendem, cada vez mais, a ocupar uma grande parte da vida contemporânea e se tornaram uma poderosa ferramenta para a criação e difusão de saberes, de opiniões e também de valores. Como Hampton et al. (2009) sugerem, cada vez mais construímos e mantemos as nossas relações sociais pelos meios de comunicação digital, o que afeta o modo como organizamos a nossa vida diária. Novas práticas socioespaciais têm surgido em um nível coletivo, como flash mobs, utilização das TIC em movimentos de reinvindicação social e política, em manifestações e protestos populares etc. (Storck, 2011). Essas práticas também surgem em um nível individual, como o uso de sistemas de navegação, recomendações para encontrar lugares interessantes para visitar, programas de compartilhamento de bicicleta e automóvel etc. A essa lista somam-se ainda inúmeras outras ações, tais como: a anotação de lugares por meio do twitter, facebook ou sites de avaliação, como Yelp; o uso de smartphones para organizar reuniões/demonstrações espontâneas; falar por meio de telefones celulares; expor temas particulares em público ou em redes sociais (por exemplo, postar fotos privadas, desejos, problemas); posicionar a voz sobre IP e videoconferência para o compartilhamento imediato de experiências e sentimentos. Esses exemplos permitem inferir acerca da emergência de novas formas de comportamento individual e coletivo, o que também se reflete na maneira como os lugares públicos são utilizados e apropriados (Graham \& Aurigi, 1997).

Deparar-se com pessoas falando ou olhando para o celular ou tablet durante uma caminhada ou uma estadia ao ar livre vem se tornando mais comum do que ver as pessoas conversando ou contemplando o espaço em que se encontram. Estudos recentes sobre tais questões têm, sobretudo, dedicado-se à análise dos aspectos mais negativos da relação entre as pessoas e o mundo virtual. 0 cyberbullying, por exemplo, pode ser mencionado como um dos recentes comportamentos de risco, sobretudo entre adolescentes, no qual novas formas de violência são exercidas com recurso do ambiente virtual da internet (Katzer, 2007, 2011; Fetchenhauer \& Belschak, 2009).

Em contrapartida, a pesquisa de Thomas (2013) sobre as conexões entre tecnologia e biofilia é especialmente interessante e inspiradora. A autora evidencia a importância da natureza para as pessoas, pois, mesmo ao imaginarmos o espaço virtual, apropriamo-nos da ecologia. Thomas identifica "technobiofilia" como a "atração inata por processos reais e da vida que aparecem em tecnologia" e, no seu blog, desafia-nos: "Será que podemos capitalizar o nosso recém-descoberto amor pela vida conectada (wired life) para encorajar mais pessoas para gozar a vida ao ar livre?" (Thomas, 2014).

Concluindo, esses fatos levantam uma pergunta importante: Como vislumbrar as TIC como um recurso que pode potencializar a qualidade ambiental, a vida pública e a participação social na produção da cidade e, em particular, do espaço público?

\section{O espaço público aberto}

No âmbito do Projeto CyberParks, entende-se espaço público aberto no seu contexto socioterritorial de cunho coletivo e no seu sentido mais amplo, isto é, como o espaço não edificado e inserido no tecido urbano, planejado, projetado e mantido com a finalidade específica e utilizado pelo público em geral. A rede de espaços públicos abertos inclui tanto os espaços naturais ou seminaturais como os espaços construídos, abrangendo, assim, ruas, praças, parques, jardins, hortas comunitárias, parques infantis etc. Cada um desses espaços desempenha um papel vital na cidade, seja para a mobilidade, vida social, lazer e recreação, seja por conta do seu mérito ambiental, como valor paisagístico e ecológico, da importância para a preservação da natureza e da paisagem, bem como da promoção da qualidade ambiental das cidades. E a par das variadíssimas mudanças societais e urbanas, paradoxalmente, esses espaços mantêm-se como proeminentes lugares de encontro, de interação social, de contato com a cidade e a natureza, preservando-se como espaços representativos do modo de vida urbano e que, como tal, são importantes no âmbito da complexa infraestrutura social (Smaniotto Costa et al., 2015). Doravante, usamos somente o termo espaço público, enquanto por produção entende-se toda a atividade relacionada com a concepção, planejamento, implementação, construção, manutenção e gestão desses espaços.

Os espaços públicos são, assim, um recurso-benefício do desenvolvimento urbano sustentável, identificando um conjunto de potencialidades, sobretudo, de âmbito cumulativo e que vão desde as questões recreacionais, socioeducacionais, econômicas, ecológicas e ambientais 
até as implicações urbanísticas (GreenKeys, 2008; Šuklje Erjavec, 2010; Smaniotto Costa, 2014, 2012). Fazendo uso de uma expressão atualmente corrente na Europa: são espaços significativos e significantes das e para as "cidades amigas das pessoas" (People friendly-city). Nesse sentido, é válido considerar que não só a existência, a distribuição e a acessibilidade a esses locais são importantes, mas também o são as questões relacionadas com a atratividade e a usabilidade desses espaços, aspectos cujas componentes do design e dos equipamentos disponibilizados têm particular influência (Šuklje Erjavec, 2010).

$\mathrm{Na}$ análise de um parque urbano, um dos mais paradigmáticos e complexos tipos de espaço público, depara-se com a necessidade de se identificarem os papéis e as funções que ele deve cumprir para se tornar parte da comunidade local. Assim, ao mesmo tempo em que um parque deve servir, por um lado, como contexto lúdico, para o uso diário e ocasional, para as diferentes camadas sociais, responder às diferentes necessidades e expectativas, proporcionar uma boa acessibilidade e um ambiente acolhedor não só no sentido físico, mas também psicológico e social, deve, por outro, também desempenhar um significativo papel na formação da imagem urbana e de representatividade sociossimbólica. Esses dois aspectos evidenciam a importância da boa integração do parque (e de qualquer outro espaço público) no tecido urbano, de um design de qualidade e propício ao uso social (Thompson \& Travlou, 2007; Thompson, 2002).

As características anteriormente citadas são relevantes para a qualificação do espaço público, já que a existência (ou não) delas interfere na escolha e no uso social do espaço. Nesse sentido, autores como Tschumi (1983), Kaplan \& Kaplan (1989), Thompson (2002), Turner (2004), Šuklje (2010) vêm sugerindo que o papel do parque urbano nas cidades contemporâneas (e nas cidades futuras) deve ser repensado de acordo com os novos estilos de vida, valores e atitudes. Porém as opiniões seguem direções diferentes e, por vezes, são conflitantes. Por exemplo, enquanto alguns autores enfatizam a experiência da visita do parque urbano para experimentar e reencontrar a natureza (Kaplan \& Kaplan, 1989; Thomas, 2014; Thompson \& Travlou, 2007), outros assinalam que devemos introduzir novos serviços que proporcionem uma variedade de programas e atividades culturais (Tschumi, 1983; Cann 1987). Com isso, um novo modelo de parque deve substituir o tradicional, como é o caso do Parc de la Villette em Paris, na França, explicado por Tschumi (1983) como park as a building, em que proporcionar espaços para atividades culturais e educacionais predomina sobre a intenção de facultar uma experiência de vivência e de aproximação da natureza na cidade (Cann, 1987).

Afinal, quais adaptações e inovações são realmente necessárias? Sendo esta uma questão fundamental para proceder a sua resposta, ainda são necessárias mais pesquisas, discussões e reflexões. Todavia, as diferentes e as divergentes opiniões indicadas relevam, acima de tudo, que as tradicionais formas de produção e de manutenção dos parques urbanos já não são suficientes para suprir as necessidades e as expectativas contemporâneas. Nesse sentido, Turner (2004) aponta que os parques urbanos vocacionados para uma lógica de people friendly devem ir além da recreação e se tornarem lugares que contribuam para saciar novas necessidades e atividades ao ar livre, ao passo que Thompson (2002) acrescenta a necessidade de novos usos e experiências nesses espaços. Como se pode verificar, a expressão novo é mencionada de forma recorrente e por uma multiplicidade de autores não só conectada aos modos de uso, mas também ao design e aos equipamentos oferecidos. No entanto, nota-se que a especificação do que, na verdade, é "novo" ou "inovação" permanece bastante difusa. Isso permite levantar a questão se, de fato, o avanço tecnológico e os novos estilos de vida vão exigir a completa reconfiguração dos espaços públicos hoje existentes. Trata-se de uma consideração que, no seu amplo sentido, quando associada às perspectivas chamadas de inteligentes (smart), nos faz questionar sobre do que se trata ou se quer tratar quando se fala de espaços públicos inovadores e inteligentes. Daqui decorrem mais algumas questões interessantes, por exemplo: Será que para algo ser inteligente precisa necessariamente estar ligado à utilização das TIC? Até que ponto um espaço público sem a interferência das TIC é mais (ou menos) atrativo para as pessoas (e para as cidades)?

Cyberpark: um espaço híbrido, locativo, pervasivo e ubíquo

A discussão inerente às inter-relações entre as TIC e as cidades vem, sobretudo, sendo conduzida por visões orientadas para o que mais recorrentemente 
está sendo designado como cidades inteligentes (smart cities). No entanto, o conceito de smart city não possui uma definição única e reconhecida. Em geral, esse é um termo coletivo e usado para enquadrar as perspectivas de desenvolvimento holístico que, atualmente, visam tornar as cidades mais eficientes, tecnologicamente mais avançadas, mais verdes e socialmente inclusivas. Por exemplo, a agenda e a política da Comunidade Europeia para as cidades inteligentes visam à melhoria de serviços e de infraestruturas por meio das TIC e abordam uma variedade de questões, incluindo mobilidade, produção, distribuição e consumo de energia, água e alimentos, saúde pública, condições ambientais e processos participativos (Hollands, 2008; European Commission 2012).

Essas visões são guiadas por perspectivas, nas quais o fenômeno técnico é a palavra-chave, e tendem a propor soluções tecnológicas para problemas complexos, porém sem capacitar as pessoas a se tornarem "cidadãos inteligentes". A vida na cidade e a experiência urbana não se reduzem apenas ao controle, eficiência e previsibilidade, mas devem também estar abertas ao confronto, ao inesperado, e lidar com diferenças. Quase que em paralelo com essas visões cresce o número de especialistas e de autores, como Bell (2011), Greenfield (2013), Klichowski et al. (2015), Söderström et al. (2014), que enfatizam a necessidade de se promover um desenvolvimento mais centrado nas pessoas, porém tirando proveito dos recursos relacionados com a utilização de novas tecnologias.

A partir de uma visão ampla e integradora, o Projeto CyberParks assume como desafio explorar as várias perspectivas que permitam melhor responder a relação entre espaço e sociedade. Desse modo, pretende-se aprofundar o conhecimento da relação entre cidade, pessoas, práticas, representações sociais e recursos (ambientais, econômicos, patrimoniais, culturais etc.), concomitante às potencialidades e às oportunidades oferecidas pelas TIC, contudo sem descuidar dos riscos que a "novidade" também pode acarretar. Assim, um dos objetivos específicos do projeto é contribuir para um melhor entendimento do que é ou pode ser um Cyberpark, que é aqui entendido como o espaço físico de uso público onde há uma intersecção com as tecnologias digitais. É evidente que essa é uma tarefa árdua e exige tenacidade e obstinação daqueles que aceitaram essa responsabilidade, pois ela pode incluir tantas facetas quanto níveis de entendimento, considerando a multiciplidade de usos e usuários, crenças, valores, representações e significados que os espaços públicos incorporam ou podem incorporar. A esse pressuposto atrela-se ainda o conceito de mídia locativa (locative media), já que o uso e a percepção do espaço estão fortemente vinculados a um espaço especifico (Souza \& Silva, 2006).

Assim, um Cyberpark constitui um novo tipo de paisagem urbana, na qual os elementos naturais e tecnológicos se misturam para gerar experiências sociais híbridas com o intuito máximo de melhorar a qualidade de vida da população. Os atributos de um Cyberpark, referenciado a partir da iniciativa de cidades inteligentes, podem ser definidos por meio da utilização de tecnologias de sensores em um espaço real acessível ao público, contribuindo, devido às tecnologias ubíquas usadas de maneiras a incrementar a sociabilidade e o compartilhamento, para a criação de um espaço em que o virtual serve para aumentar a paisagem real. Nesse contexto, as TIC podem ser utilizadas para fornecer ou obter informações, tornando-se um meio de interação para ajudar na produção de espaços, permitir o crowdsourcing de informações e de opiniões e ainda possibilitar o compartilhamento ou o (auto)monitoramento de atividades.

Os exemplos de hardwares incorporados ao espaço real vão desde sistemas sensíveis de som ou iluminação até sistemas de controle e vigilância, objetos cinéticos ou obras de arte e tecnologias de sensores passivos a sistemas de visualização, reconhecendo também que o uso e a percepção de tais funcionalidades diferem de acordo com as horas do dia, estações do ano, duração da estadia, clima e temperatura, localização, características da cultura urbana, engajamento individual ou de um determinado grupo social, idade e sexo dos utilizadores, propósito da visita, da topologia e tamanho do espaço.

Potencial das novas tecnologias:

alguns exemplos

Rede wi-fi em espaços públicos: oferecer gratuitamente acesso sem fios à rede de internet em espaços públicos está se tornando um serviço-padrão em muitas cidades. E talvez também para cidades mais inclusivas, já que, ao permitir a acessibilidade 
a mais habitantes, desde que disponham de dispositivos adequados, contribui também para um acesso mais democrático às infraestruturas e aos serviços urbanos. Oferecida, a princípio, por empresas privadas, observa-se a tendência para um maior envolvimento do poder público na ampliação dessa atividade - e em muitas cidades a infraestrutura digital já é um importante serviço público. A lista de cidades que oferece esse serviço em espaço público é vasta e crescente, tais como Barcelona (Espanha), Curitiba (Brasil), Lisboa (Portugal), Nova Iorque (Estados Unidos), Paris (França), que testemunham a imersão de novas tecnologias nos espaços públicos; aliás, uma das mais comuns situações de interação entre espaço real e virtual (Figura 1).

A oferta de serviço wi-fi em espaço público também tem contribuído para o surgimento de novos tipos de mobiliário urbano, projetados para facilitar o uso da rede wi-fi, como em painéis informativos sobre a disponibilidade de rede a novos tipos de mobiliário, que é o caso dos BLOCPARC instalados em Paris (França). Nesse contexto, a capital francesa testa, atualmente, seis diferentes tipos de mobiliário urbano inteligentes, entre eles o Porto Digital (Digital Harbour), uma espécie de quiosque aberto com um telhado verde, com mesas e assentos giratórios projetados para o uso de computadores portáteis, oferecendo acesso gratuito ao wi-fi e a pontos de recarga de dispositivos eletrônicos (Blocparc, 2015).

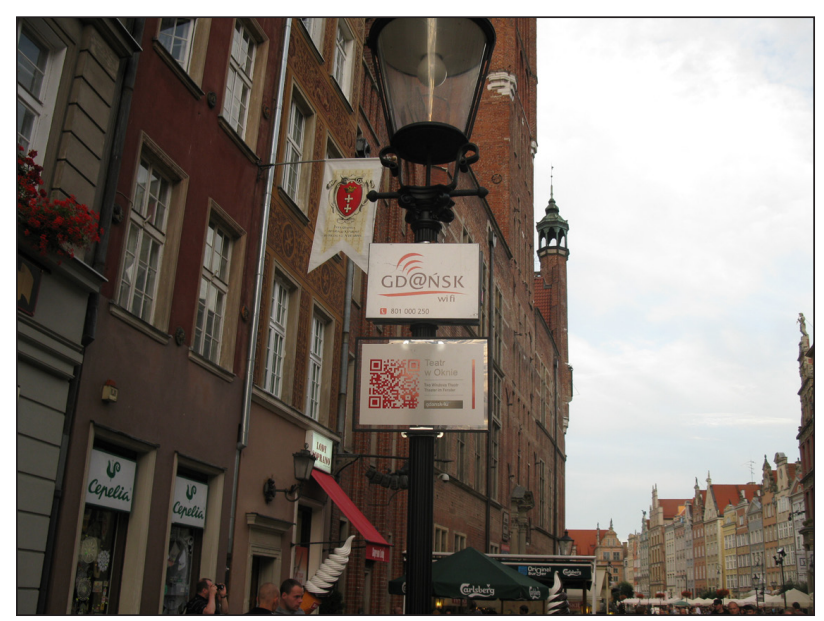

Figura 1 - Para 0 campeonato europeu de futebol de 2012, as cidades-sede da Polônia instalaram vários hotspots para acesso gratuito à internet em espaccos públicos. Placas sinalizadoras indicavam as áreas de cobertura, como na cidade de Gdansk, onde, por meio dos códigos QR, obtinha-se informaç̃õo turística sobre esses lugares Fonte: Smaniotto Costa (2012).
Equipamentos e componentes urbanos: diferentes equipamentos digitais, sensores, aplicativos e jogos podem ser usados como parte de uma estratégia ou programa para melhorar a atratividade do espaço público. Ao incorporar novos equipamentos ou peças de mobiliário urbano - como mapas interativos ou o Digital Harbour mencionado - para que as pessoas estejam digitalmente conectadas ao ar livre, paralelamente contribui, por meio de novas formas de intervenção, para acrescentar valor aos espaços públicos, aumentando a atratividade deles, mas também incrementando o uso social por intermédio do desenvolvimento de novas atividades.

Subjacente a essa perspectiva de incremento das atividades em espaço público pela promoção do contato com ambientes ao ar livre, idealmente, pode-se estimular o desenvolvimento de estilos de vida mais ativos e saudáveis. Nesse sentido, o desenvolvimento de experiências como os "urban games" e os "Geocaching" (Smaniotto Costa \& Steinmeier, 2012) podem ajudar a desenvolver ambientes mais atraentes e interativos, apoiados por diferentes programas e eventos culturais, artísticos, educacionais e de lazer. Em Ljubljana (Eslovênia), por exemplo, o Parque Severni Mestni foi projetado para atrair uma ampla gama de utilizadores, desde aqueles mais habituais aos que são visitantes ocasionais, recorrendo a portais multimídia que oferecem a possiblidade de aprender, experimentar e interagir sobre cultura e arte, especialmente sobre literatura (Figura 2).

Reforçar a dimensão pública: a capacidade das TIC para melhorar a comunicação entre (potenciais) usuários cria possiblidades de participação criativa e de capacitação social, contribuindo para que as pessoas possam melhor articular os seus interesses. Além das TIC serem uma importante ferramenta para a construção de cenários e de simulações, quando usadas para a comunicação social podem ajudar a reforçar a atratividade, a divulgação de informação sobre o próprio espaço, os seus equipamentos e serviços, bem como sobre as atividades desenvolvidas em espaços públicos específicos. Essa interação entre utilizadores do espaço e das TIC pode também contribuir para o compartilhamento de informações, a exposição de opiniões, de necessidades e de desejos relativamente ao espaço público, acrescentando 


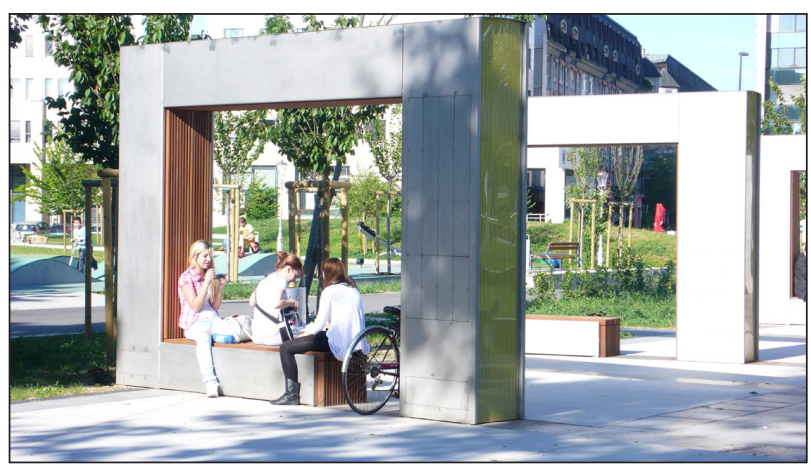

Figura 2 - Os portais multimídia instalados no Parque Severni Mestni (Liubliana, na Eslovênia) possibilitam a leitura e a escuta de clássicos da literatura internacional na linguagem original. Portais USB e caixas de som instaladas permitem também escutar músicas próprias com volume limitado Fonte: In.Ka.Bi (2012).

informação àqueles mais diretamente envolvidos com a produção dos espaços públicos. Assim, os dispositivos de GPS ou SIG também ajudam a melhor interpretar e compreender as relações entre utilizadores e espaço.

\section{Aprofundar a compreensão e o conhecimento:} as novas tecnologias, suas aplicações e dispositivos também estão permitindo uma variada gama de oportunidades para melhor compreensão dos contextos, para o compartilhamento de informação e do conhecimento, permitindo a descoberta e o apoderamento da cidade. Exemplos vão desde sensores que possibilitam a captação de dados até mapas interativos e novas formas de intercâmbio entre usuários e espaços. Como exemplos práticos podemos citar as estratégias da cidade britânica de Bristol legible city ou playable city, onde a prefeitura se valeu das TIC para melhorar a legibilidade e a habitabilidade da cidade e permitir o uso lúdico de todo o espaço urbano (Bristol City Council, 2015). Outro exemplo se verifica por meio de jogos criativos, como os urban games (jogos urbanos), que, ao exigirem o engajamento de pessoas com a cidade real, apoiam um modo de vida mais saudável, como The Mobile City em Bristol, Inglaterra (Watershed, 2015) ou Hackble City em Amsterdã, Holanda (Mobile City, 2015).

Existem também uma gama diferenciada de aplicativos que identificam plantas e animais, como é o caso dos aplicativos Bird Song Id, que identifica espécies de pássaros a partir de sons captados pelo microfone do dispositivo (Isoperla, 2015), e o PlantNet, que ajuda a identificar uma planta ao comparar as semelhanças visuais com as existentes no banco de imagens (PlantNet, 2015). Embora tais experiências possam também ser alcançadas por meio de elementos convencionais, provavelmente recorrer a aplicativos ou a mobiliários digitais seja interessante elemento de atratividade social e de fomento para o uso do espaço público.

0 apoio à pesquisa: as novas tecnologias digitais abrem oportunidades inovadoras de/e para a investigação. Como uma ferramenta interativa, permite o contato com usuários, bem como possibilita o envio de opiniões e de sugestões. Nesse sentido, o Projeto CyberParks está desenvolvendo um aplicativo, designado como WAY CyberParks, capaz de coletar informações sobre o comportamento das pessoas, suas necessidades, preferências e motivações no uso de espaços públicos. Atualmente, o aplicativo está sendo testado em Barcelona e Bilbao (Espanha), Bristol (Inglaterra), Lisboa (Portugal) e Ljubljana (Eslovênia), porém a sua extensão a outras cidades é fácil de ser organizada.

O WAY CyberParks consiste de três elementos básicos: um aplicativo para smartphones (app), um conjunto de serviços web e um de nuvem, que contribuem para a implementação de várias funções, e a mais relevante para este trabalho é o monitoramento de usuários. Toda vez que um usuário se encontra na área de estudo, o referido app gera sinais em intervalos predeterminados que permitem observar no serviço web os seus movimentos e, assim, visualizar itinerário percorrido, o tempo de permanência em determinados locais etc (Figura 3). Outra funcionalidade importante é o recurso da realidade aumentada, que permite a obtenção de informações contextuais, possibilitando ainda o envio de sugestões ou de queixas (Figura 4), ou responder a questionários. Sempre que um usuário se aproxima de áreas definidas, o aplicativo lança questões pré-elaboradas. Ao acionar o app no smartphone, é pedido aos participantes que definam seu perfil (sexo, idade, instrução e ocupação). A soma desses perfis permite a elaboração de uma matriz de usuários para cada área de estudo. Toda a informação recolhida - perfil dos usuários, posições em tempo 


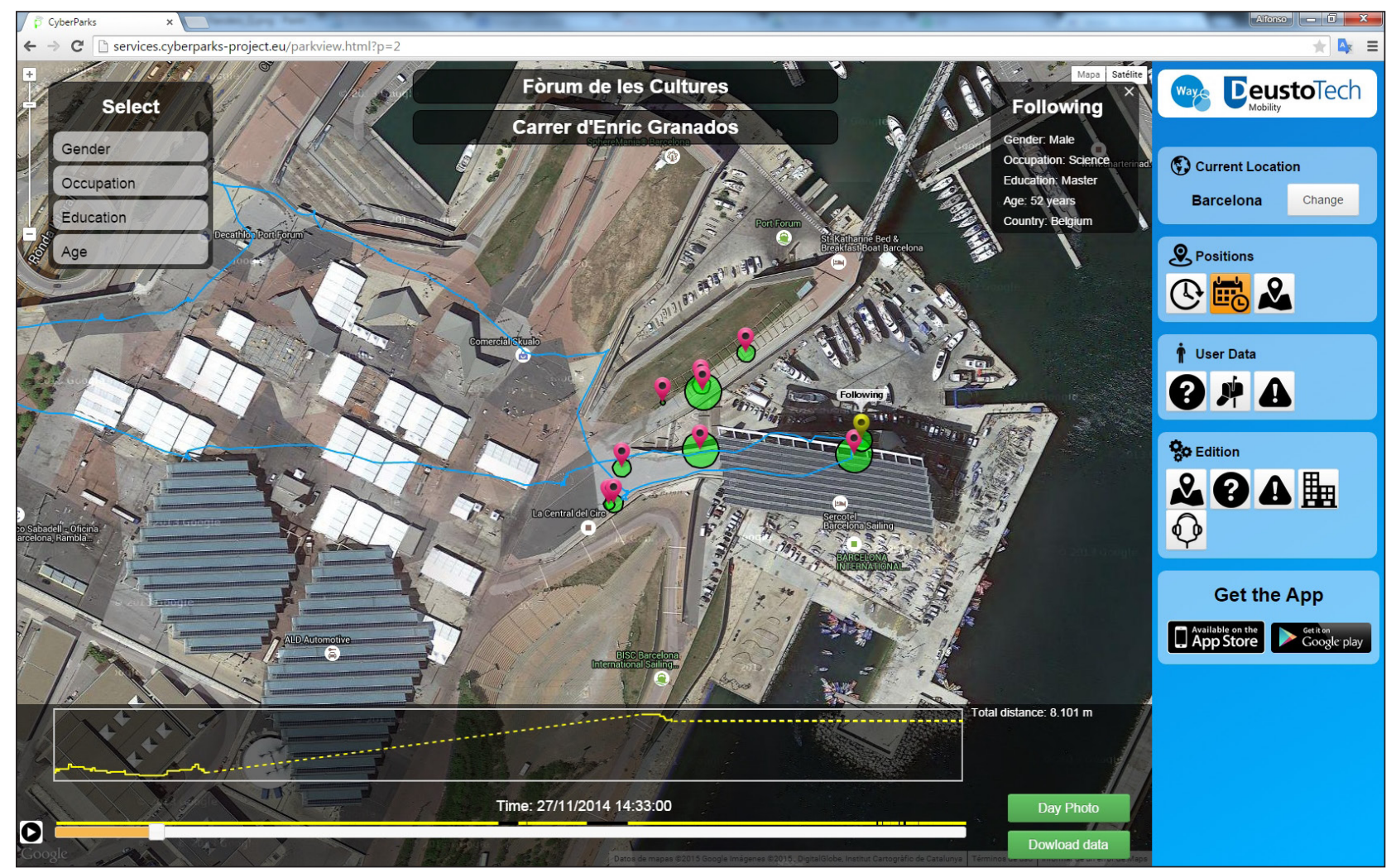

Figura 3 - Imagem do serviço web do aplicativo WAY CyberParks mostrando o itinerário de um usuário no "Fòrum de les Cultures", em Barcelona (Espanha). Os círculos verdes indicam a incidência de usuários Fonte: CyberParks (2015).

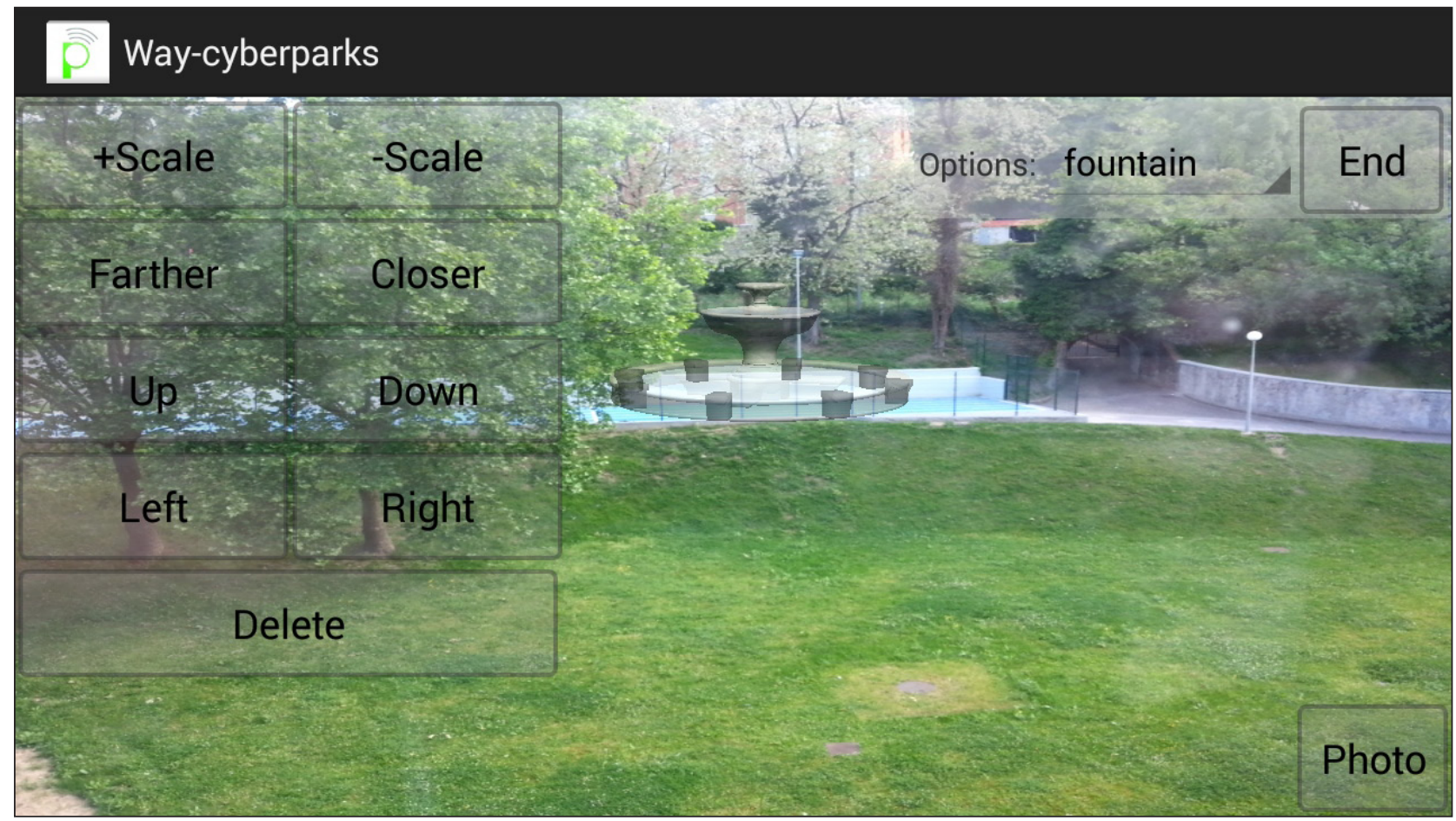

Figura 4 - 0 aplicativo WAY CyberParks abre novas oportunidades de interação com usuários, por exemplo, a o oferecer a oportunidade de fazer comentários ou subir fotos do espaço visitado Fonte: CyberParks (2015). 
real, respostas e sugestões - é enviada e armazenada na nuvem, a partir da qual serviços web buscam as informações. Os serviços web permitem visualizar os dados recolhidos e cruzá-los com outras informações relevantes, como as condições meteorológicas e perfil do usuário. Toda a informação recolhida pode ser visualizada com base no Google Maps, em tempo real e/ou por meio do histórico, com possibilidade de análise dos diferentes itinerários, bem como de dados gerais relacionados com o perfil social do utilizador. O sistema opera em dois modos: on-line e off-line. No primeiro modo, o aplicativo envia continuamente para a nuvem dados (perfil e posição) por meio de um serviço de comunicação ativo (GPRS, 3/4G ou Wi-Fi). No modo off-line, o aplicativo salva os dados na memória do smartphone e os envia para a nuvem quando o usuário permitir, por exemplo, ao chegar a um lugar com acesso à internet. 0 aplicativo WAY CyberParks está disponível nos consoles do Google Play (Android), na Apple Store (iOS), bem como no site do projeto.

\section{O caso de estudo de Lisboa e notas finais}

Um importante aspecto das tecnologias digitais é possibilitar o diálogo entre espaços e usuários, bem como entre usuários e aqueles que se interessam pela produção dos espaços públicos. Em Lisboa (Portugal), desenvolver esse diálogo é um ponto central do projeto. Dois testes iniciais, e em dois diferentes parques da cidade, Jardim da Estrela e Parque da Quinta das Conchas (ver Smaniotto Costa, 2012), foram realizados em junho de 2014 e 2015. Os testes foram acompanhados de um questionário, $\mathrm{e}$ em cada parque recorreu-se a um dispositivo diferente. No Jardim da Estrela, um parque implementado no fim do século XVIII e inserido no coração histórico da cidade, foram usados dispositivos de GPS com o objetivo de rastrear o itinerário de visitantes (Figura 5). 0 aplicativo WAY CyberParks foi utilizado no Parque da Quinta das Conchas, um parque instalado em 2005 no remanescente de uma quinta e agora inserido em uma área de adensamento e de expansão urbana. Os resultados da análise, ainda que preliminares, estão discutidos em Smaniotto Costa et al. (2014),

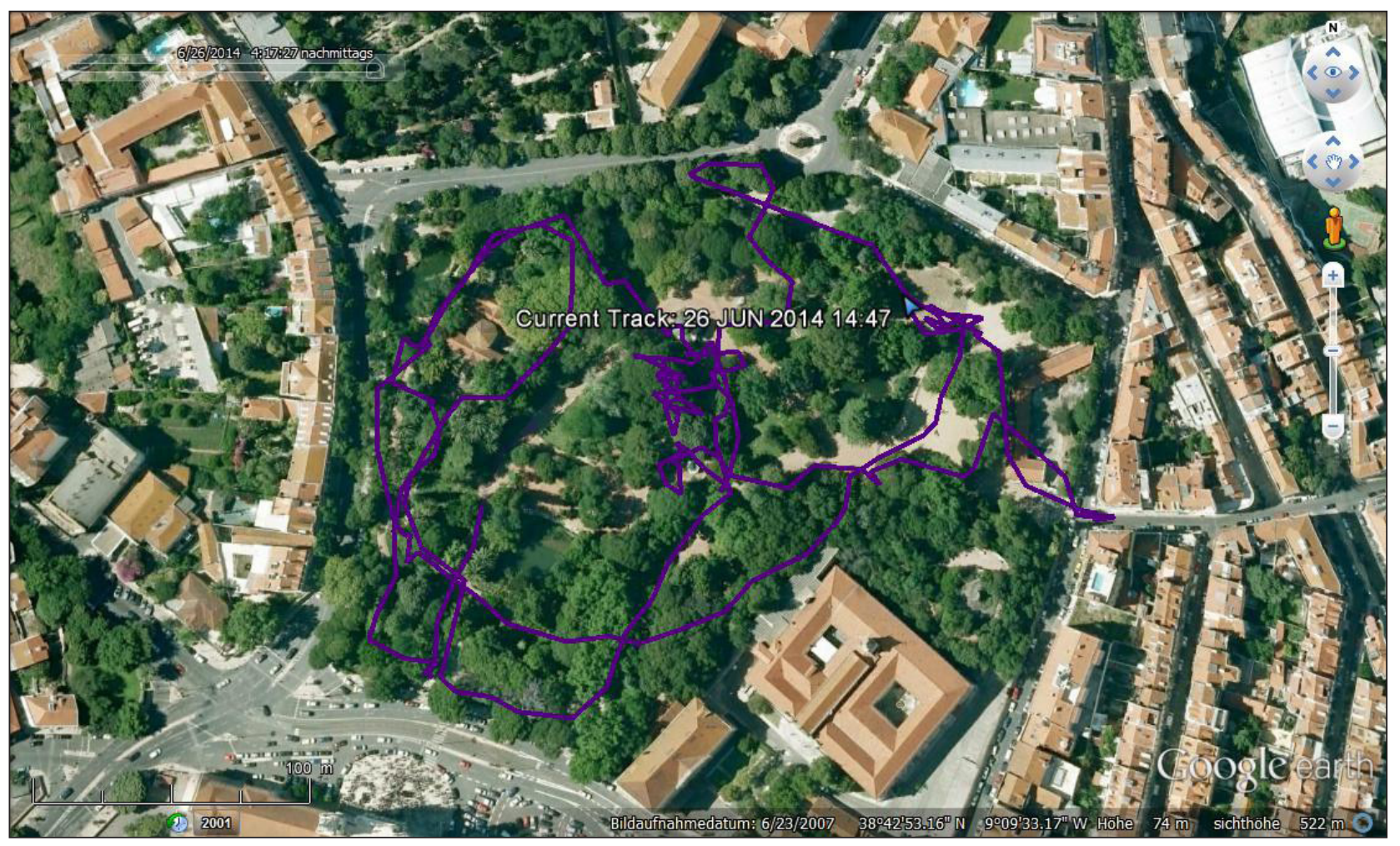

Figura 5 - A representação típica de um itinerário no Jardim da Estrela (Lisboa, em Portugal), com o trajeto e os pontos. Para cada ponto, além das coordenadas, foi registrado o tempo em que foi alcançado Fonte: CyberParks (2015). 
nos quais também se discute a aplicabilidade das ferramentas tecnológicas utilizadas.

0 recurso a essas ferramentas no processo de investigação é uma tentativa de utilizar as TIC para melhor perceber como os espaços são usados. Assim, inicia-se um caminho de pesquisa que visa responder a um objetivo mais amplo: contribuir, por meio do conhecimento científico, para a criação e o planejamento de espaços públicos urbanos mais atrativos, inclusivos e seguros.

Nos estudos realizados em Lisboa (Portugal), observou-se, a partir da comparação entre itinerário dos usuários e as características locais, a confirmação de uma tendência conhecida: onde há atrativos, o ambiente é agradável, convidativo, e oferece diferentes possibilidades de apropriação, e as pessoas tendem a frequentar mais e despender mais tempo. Isso pode contribuir para uma maior convivência social com todas as suas derivantes, tais como identificação, sentido de pertencimento, interação social etc.

Uma outra conclusão a que se chega é que o recurso às ferramentas tecnológicas - que são uma mais-valia para a investigação e para o planejamento - não deve ser realizado de forma que se neguem as técnicas e as metodologias de investigação e de planejamento mais tradicionais (Menezes, 2012). Aqui, o desafio está em encontrar complementaridades entre perspectivas teórico-metodológicas e técnicas tradicionais e mais inovadoras, de modo a incrementar o uso dos espaços públicos urbanos por meio de um planejamento inteligente e inclusivo. Porém essas possibilidades são aqui assumidas como um desafio e, de modo algum, restringem-se a uma simples adição da tecnologia ao ambiente urbano, isto é, as TIC são aqui vistas como um elemento de criação de espaços mais atraentes e interativos, contribuindo para a agregação de valor aos espaços reais.

Todavia, ainda que a existência de espaços públicos seja reconhecida e valorizada, bem como seus atributos para aumentar a qualidade de vida urbana, o que se observa é que, em muitas cidades, as potenciais funções e qualidades dos espaços públicos não são exploradas e muitas vezes são sacrificadas em benefício do tráfego motorizado (Smaniotto Costa, 2014). Na verdade, a promoção de espaços públicos de qualidade nem sempre é uma prioridade da política urbana. Ainda assim, muito embora o Projeto CyberParks esteja orientado para a sociedade europeia, a atualidade e a pertinência do tema permitem estender a problemática de estudo a outras e diferentes realidades urbanas. O Projeto CyberParks busca ampliar o sentido e o significado público das questões espaciais, socioambientais e agora tecnológicas - aliás, questões que tanto influenciam em nossas vidas. Desse modo, julga-se que as novas tecnologias, se bem pensadas e adaptadas, podem ser um importante recurso-benefício para a promoção do direito a um espaço urbano de qualidade.

Os estudos realizados, ainda que incipientes, demonstram que o espaço público não se tornou supérfluo, nem tampouco o mundo virtual se tornou um substituto do real. Os avanços do Projeto CyberParks têm permitido constatar que os espaços - real e virtual -complementam-se. E nada mais inteligente do que tirar proveito dessa intersecção e melhorar a qualidade dos espaços reais, para que eles continuem como palco e âncora do mundo virtual, e vice-versa.

\section{Referências}

Bell, S. (2011). System city: urban amplification and inefficient engineering. In M. Gandy (Ed.), Urban constellations (pp. 72-74). Berlin: Jovis.

Blocparc. (2015). Recuperado em 01 de julho de 2015, de www.blocparc.fr

Bristol City Council. (2015). Bristol legible city. Bristol. Recuperado em 01 de julho de 2015, de www.bristollegiblecity. info/initiative.htm

Cann, B. (1987). The park of la villette: urban park as building. Places, 4(3), 217-220.

Cost Framework. (2015). TUD COST Action TU1306. Brussels: Cost. Recuperado em 01 de julho de 2015, www.cost.eu/ domains_actions/tud/Actions/TU1306

CyberParks. (2015). The applicacation WAY CyberParks. Lisboa: CyberParks. Recuperado em 01 de julho de 2015, de http://cyberparks-project.eu/app

European Commission. (2012). European initiative on smart cities. Recuperado em 01 de julho de 2015, de https://setis.ec.europa.eu/set-plan-implementation/ technology-roadmaps/european-initiative-smart-cities

Fetchenhauer, D., \& Belschak, F. (2009). Cyberbullying in chatrooms: who are the victims? Journal of Media Psychology, 21(1), 25-36. http://dx.doi.org/10.1027/1864-1105.21.1.25. 
Godbey, G. (2009). Outdoor recreation, health, and wellness: understanding and enhancing the relationship. Washington: Resources for the Future. Recuperado em 15 de maio de 2014, de www.rff.org/files/sharepoint/WorkImages/ Download/RFF-DP-09-21.pdf

Graham, S., \& Aurigi, A. (1997). Virtual cities, social polarisation and the crisis in urban public space. Journal of Urban Technology, 4(1), 19-52. http://dx.doi. org/10.1080/10630739708724546.

Greenfield, A. (2013). Against the smart city (the city is here for you to use Book 1). New York: Do Projects.

GreenKeys. (2008). GreenKeys @your city: a guide for urban green quality. Dresden: IOER. Recuperado em 15 de maio de 2014, de www.greenkeys-project.net

Hampton, K., Livio, O., \& Sessions, L. (2009). The social life of wireless urban spaces: internet use, social networks, and the public realm. Journal of Communication, 60(4), 701-722. http://dx.doi.org/10.1111/j.1460-2466.2010.01510.x.

Hollands, R. G. (2008). Will the real smart city please stand up? Intelligent, progressive or entrepreneurial? City, 12(3), 303-320. http://dx.doi.org/10.1080/13604810802479126.

Isoperla. (2015). Wildlife app: bird song id. Recuperado em 10 de julho de 2015, de www.isoperlaapps.com

Kaplan, R., \& Kaplan, S. (1989). Experience of nature: a psychological perspective. New York: Cambridge University Press.

Katzer, C. (2007). Gefahr aus dem Netz-Der Internet-Chatroom als neuer Tatort für Bullying und sexuelle Viktimisierung von Kindern und Jugendlichen (Dissertação de mestrado). Universität Köln, Köln.

Katzer, C. (2011). Das Internet als Tatort: Cyberbullying und sexuelle Gewalt- Wer sind die Täter, wer wird zu Opfern? Hannover: Landesstelle Jugendschutz Niedersachsen.

Klichowski, M., Bonanno, P., Jaskulska, S., Smaniotto Costa, C., de Lange, M., \& Klauser, F. (2015). CyberParks as a new context for Smart Education: theoretical background, assumptions, and pre-service teachers' rating. American Journal of Education, 3(12A), 1-10.

Menezes, M. (2012). L'espace du social dans un monde de (multi)représentations socio-spatiales: meta-réflexion méthodologique à partir d'un regard géo-anthropologique . In C. Cerrti, I. Dumont \& M. Tabusi (Eds.), Geografia sociale e democrazia: la sfida della communicazione (pp. 87-94). Roma: Aracne Editrice.
Mobile City. (2015). Mobile media and urban design. The Hackable City. Recuperado em 10 de julho de 2015, de http://themobilecity.nl/2013/amsterdam

PlantNet. (2015). Plant observation and interactive identification. Recuperado em 10 de julho de 2015, de www. plantnet-project.org

Smaniotto Costa, C. (2012). De quintas a parques. Visitando os Parques da Quinta das Conchas e da Quinta dos Lilases em Lisboa. Arquitextos, 1-11. Recuperado em 16 de abril de 2015, de www.vitruvius.com.br/revistas/read/ arquitextos/13.146/4429

Smaniotto Costa, C. (2014). Can we change processes in our cities? Reflections on the role of urban mobility in strengthening sustainable green infrastructures. Journal of Traffic and Logistics Engineering, 2, 141-155.

Smaniotto Costa, C., \& Schmitz, R. M. (2013). As modernas Tecnologias de Informação e Comunicação e o espaço público: explorando as fronteiras de uma nova relação. Revista de Geografia e Ordenamento do Território, 3, 197-229.

Smaniotto Costa, C., \& Steinmeier, G. (2012). A caça ao tesouro ao ar livre. Geocaching, uma oportunidade de lazer em espaços verdes. Arquitextos, 12, p. 143. Recuperado em 16 de abril de 2015, de www.vitruvius.com.br/revistas/ read/arquitextos/12.143/4332

Smaniotto Costa, C., Menezes, M., \& Mateus, D. (2014). How would tourists use Green Spaces? Case Studies in Lisbon. Entretextos, 52, 29. Recuperado em 16 de abril de 2015, de www.ceied.ulusofona.pt/pt/investigacao/publicacoes/ entretextos

Smaniotto Costa, C., Menezes, M., Mateus, D., \& Martinez, A. B. (2015). As tecnologias da informação-comunicação contribuem para conhecer as práticas e necessidades sociais em parques urbanos? 0 projeto COST-Action CyberParks-TU 1306. In Anais do XII CONLAB - Congresso Luso-Afro-Basileiro de Ciências Sociais (pp. 7705-7713). Casal de Cambra: Caleidoscópio.

Söderström, O., Paasche, T., \& Klauser, F. (2014). Smart cities as corporate storytelling. City, 18(3), 307-320. http://dx.doi. org/10.1080/13604813.2014.906716.

Souza \& Silva, A. (2006). From cyber to hybrid: mobile technologies as interfaces of hybrid spaces. Space and Culture, 9(3), 261-278. http://dx.doi.org/10.1177/1206331206289022.

Storck, M. (2011). The role of social media in political mobilisation: a case study of the january 2011 Egyptian uprising. Recuperado em 22 de abril de 2014, de http:// de.scribd.com/doc/132230246 
Šuklje Erjavec, I. (2010). Designing an urban park as a contemporary user-friendly place. In B. Golicnik Marušić \& M. Nikšič (Eds.), Human cities: celebrating public space (pp. 39-51). Oostkamp: Stichting Kunstboek.

Thomas, S. (2013). Technobiophilia: nature and cyberspace. London: Bloomsbury.

Thomas, S. (2014). Cyberparks will be intelligent spaces embedded with sensors and computers. Boston: The Conversation. Recuperado em 15 de abril de 2015 de http://theconversation.com/cyberparks-will-be-intelligentspaces-embedded-with-sensors-and-computers-26837

Thompson, C. W. (2002). Urban open space in the 21st century. Landscape and Urban Planning, 60(2), 59-72. http://dx.doi.org/10.1016/S0169-2046(02)00059-2.
Thompson, C. W., \& Travlou, P., editors. (2007). Open space: people space. Abingdon: Routledge.

Tschumi, B. (1983). Parc de la Villette. Paris: A.D.A. Edita.

Turner, M. (2004). Urban parks as partners in youth development. In The Urban Institute. Beyond recreation: a broader view of urban park (pp. 1-7). New York: The Wallace Foundation.

Watershed. (2015). The playable city. Recuperado em 01 de julho de 2015, de www.watershed.co.uk/playablecity/ overview

Recebido: Set. 14, 2015

Aprovado: Out. 12, 2015 